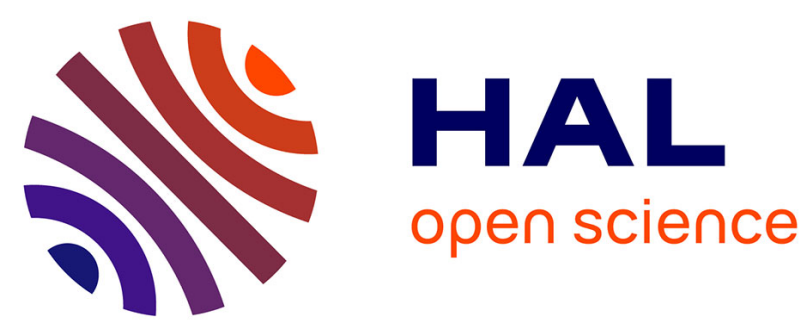

\title{
Narrow energy distributions of electrons emitted from clean graphene edges
}

R. Diehl, M. Choueib, S. Choubak, R. Martel, S. Perisanu, Anthony Ayari, P. Vincent, S. Purcell, P. Poncharal

\section{- To cite this version:}

R. Diehl, M. Choueib, S. Choubak, R. Martel, S. Perisanu, et al.. Narrow energy distributions of electrons emitted from clean graphene edges. Physical Review B: Condensed Matter (1978-1997), 2020, 102 (3), pp.035416. 10.1103/PhysRevB.102.035416 . hal-02905047

\section{HAL Id: hal-02905047 \\ https://hal.science/hal-02905047}

Submitted on 5 Nov 2020

HAL is a multi-disciplinary open access archive for the deposit and dissemination of scientific research documents, whether they are published or not. The documents may come from teaching and research institutions in France or abroad, or from public or private research centers.
L'archive ouverte pluridisciplinaire HAL, est destinée au dépôt et à la diffusion de documents scientifiques de niveau recherche, publiés ou non, émanant des établissements d'enseignement et de recherche français ou étrangers, des laboratoires publics ou privés. 


\title{
Narrow energy distributions of electrons emitted from clean graphene edges
}

\author{
R. Diehl, ${ }^{1}$ M. Choueib, ${ }^{2}$ S. Choubak,${ }^{3}$ R. Martel, ${ }^{3}$ S. Perisanu, ${ }^{2}$ A. Ayari $\odot,{ }^{2}$ P. Vincent, ${ }^{2}$ S. T. Purcell,,${ }^{2}$ and P. Poncharal $\oplus^{2, *}$ \\ ${ }^{1}$ Gloor Instruments AG, Schaffhauserstrasse 121, 8302 Kloten, Switzerland \\ ${ }^{2}$ Institut Lumiére Matière (UMR CNRS 5306), University of Lyon, Université Claude Bernard, 69622 Villeurbanne, France \\ ${ }^{3}$ Département de Chimie, 2900, Boulevard Edouard-Montpetit, Montréal (Québec) H3T 1J4, Canada
}

(Received 19 February 2020; accepted 18 June 2020; published 10 July 2020)

\begin{abstract}
This paper presents a study on electron field emission (FE) into ultrahigh vacuum (UHV) from a single, cleaned, graphene sheet where its single sheet nature has been carefully characterized and the emission zones are unambiguously the sheet edges. This definitive approach is in contrast to almost all other studies in the literature and can now guide theoretical work and applications. Our sample characterization starts with transmission electron microcopy imaging, Raman characterization, controlled mounting of individual flakes in a scanning electron microscope followed by in situ high temperature and high field treatments. Detailed FE characterization was carried out including current-voltage plots, FE microscopy, and electron energy spectroscopy during subsequent stages of sample cleaning, at room and liquid air temperatures. The full width at half maximum (FWHM) of the energy peak as well as its relative shift versus current and voltage were explored. A notable result is that the energy spectra are very narrow, ten times less than previously reported.
\end{abstract}

DOI: 10.1103/PhysRevB.102.035416

Graphene, a subject of great interest for many domains of science and technology, has logically been considered for field emission (FE) sources simply because its single atomic edge has potentially nature's highest enhancement of electric field, thus opening perspectives for low voltage vacuum nanoelectronics. Though considerable work has been done [1-15] there is a dearth of measurements on well-characterized, individual single sheet graphene layers that can guide the community towards future developments. The published work is primarily on multiemitter films or individual multilayer emitters (see Ref. [16] for a review) with a few clear proofs of single layer edge emission and only one other example of ultrahigh vacuum (UHV) cleaning (on multilayer sample) which is necessary for controlled field emission studies.

A first work succeeded in obtaining FE from the edge of an exfoliated single layer [9], whose nature was proven by Raman spectroscopy, using nanomanipulation tools in a scanning electron microscope (SEM) where a deposited layer was scratched by a probe tip to dress an edge somewhat vertically. Stable currents and I/Vs were obtained which had novel upward curving Fowler Nordheim (FN) plots. Mechanical manipulation was used in an optical microscope to place what was probably a graphene flake on a tip that was not visible with the optical resolution [15] though Raman spectroscopy supported that the object contained some single layer material. Very unstable currents were obtained. Multilayer samples with gradual current conditioning cleaning were studied at the atomic level in a TEM [10] and in an UHV environment with sample heating [11] as in this work. Significant and original FEM patterns in the form of a large band composed of finer transverse elliptical forms were obtained as discussed below.

*Corresponding author: philippe.poncharal@univ-lyon1.fr
The total energy distributions (TEDs) of the emitted electrons are of particular interest because they give access to the density of states near the Fermi level and thus of the graphene edge and corner states which is a very active research domain $[17,18]$. Only two measurements [12,13] of the energy distributions of emitted electrons from uncleaned edges covered with multilayer graphene have been reported. In Ref. [13] peak widths of $\sim 2 \mathrm{eV}$ were shown that shifted to lower energy with increasing applied field. On the theoretical side there is an increasing body of work on FE from graphene edges [19-25] and reference therein of which two predict the energy distribution [19,20]. Zigzag clean edges are predicted to have well defined peaks at the Fermi level while clean armchair edges have predicted shifts of $0.5 \mathrm{eV}$ to $2 \mathrm{eV}$ below the Fermi level. Surprisingly Ref. [19] predicts peaks of $1 \mathrm{eV}$ width and Ref. [20] predicts widths of $<0.1 \mathrm{eV}$ (estimated). Peak widths for cold FE from metallic emitters are in the $0.25-0.4 \mathrm{eV}$ range [25]. Obviously this needs clarification and is a major point of this paper. From a more practical point of view TEDs can also be used to measure the voltage drops along a sample as well as to estimate the temperature of the emitting zone [26,27].

Graphene was produced by chemical vapor deposition (CVD) with a strict control of hydrogen etching to achieve a coverage with mostly single layer graphene with some bilayer islands [28,29] and transferred to transmission electron microscopy (TEM) grids. Graphene flakes were torn from the layers and picked up at the apex of etched tungsten tips (see Fig. 1). Complementary Raman spectroscopy and TEM diffraction measurements were performed on the very same grid sample. These Raman measurements (see Fig. 2) show that our sample was indeed graphene, i.e., small D band and single sharp 2D band. Although a single 2D band cannot rule out turbostratic stacking [30], we clearly can exclude graphitelike multilayered material. The TEM diffraction (see 


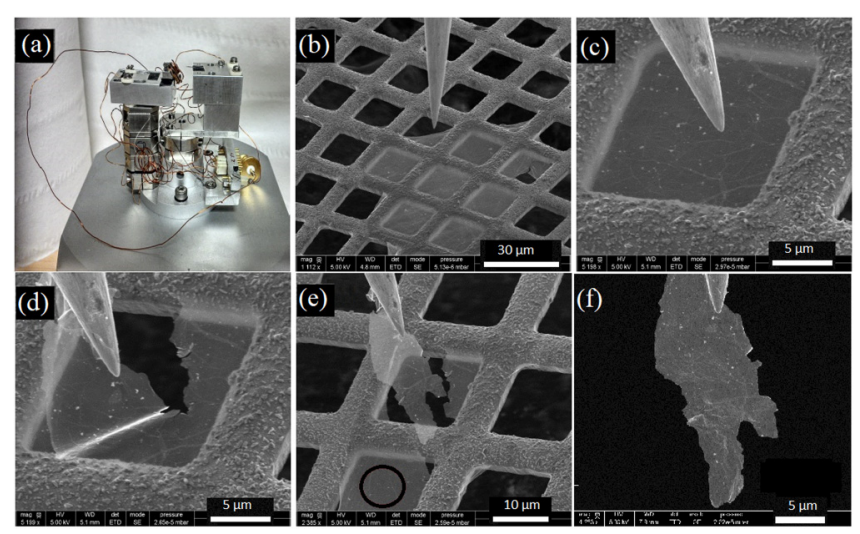

FIG. 1. Experimental setup for graphene sample production. (a) Three-axis nanomanipulator installed in our SEM. (b)-(e) Pickup sequence of a graphene flake, starting from CVD grown graphene on a TEM grid to finish with a graphene flake on a tip. The black circle shows the location of the TEM imaging analysis (see later in the text). (f) The graphene sample studied in this paper, nicknamed "Italy."

Fig. 3) shows that the sample is however polycrystalline with small single crystal grain size of on average a few tens of nms. TEM diffraction on a single grain shows the hexagonal pattern. These tips were then mounted in a polyvalent field emission system with a base pressure of $1 \times 10^{-10}$ Torr, sample heating by a W loop, imaging by microchannel plate for FE microscopy and an electron energy analyzer (see Fig. 4).

For metallic and semimetallic emitters, the IV dependence is described well by Fowler-Nordheim (F.-N.) theory [31], which predicts that the emitted current density $(J)$ versus electric field $(F)$ follows an exponential law $J(F)=$ ct $_{1} \cdot F^{2} \cdot \exp \left(-c t e_{2} . v(F) \cdot \frac{\Phi^{3 / 2}}{F}\right)$, where $c t e_{1} \sim 1.54110^{-6} \frac{A \cdot e V}{V^{2}}$, cte $e_{2}=\sim 6.83 \mathrm{eV}^{-\frac{3}{2}} \mathrm{Vnm}^{-1}$, and $v(F)$ is a function of the

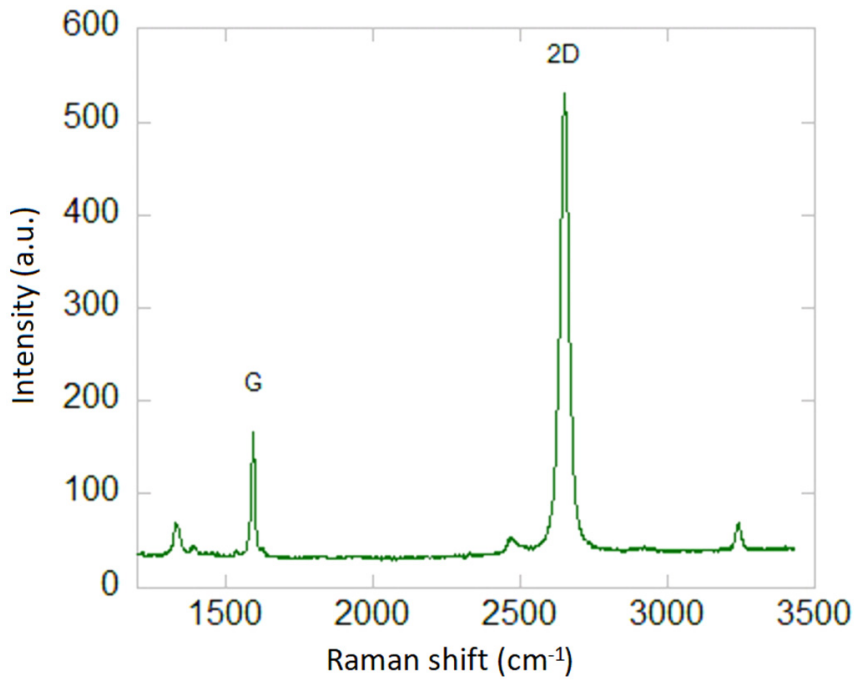

FIG. 2. Raman spectra of our graphene batch sample exhibiting a sharp single $2 \mathrm{D}$ peak in contrast to multiple peaks observed with Bernal staked multilayer.
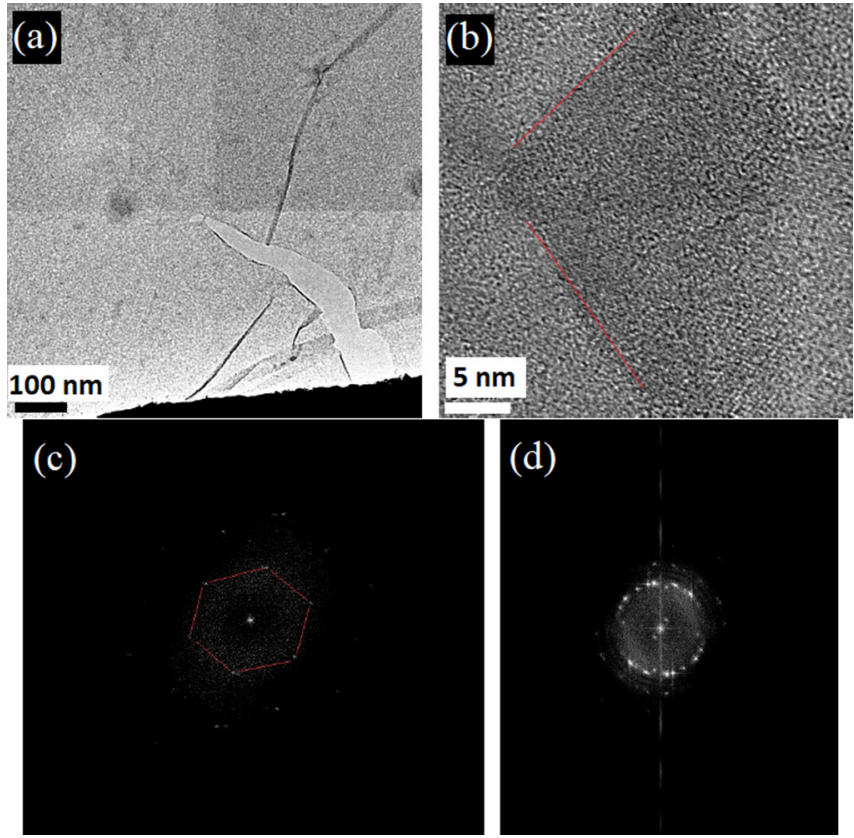

FIG. 3. TEM study of our sample. (a) Low magnification, (b) high resolution of the interest area, with visible crystal boundary. (c) Diffraction pattern of (b) showing hexagonal pattern, (d) diffraction pattern of a larger area showing the multigrain crystal structure of our graphene flake.

local electric field $\left[v(F)=1-F+\left(\frac{1}{6}\right) F \cdot \ln (F)+\ldots\right]$ [32]. Plotted on a logarithmic scale $\ln \left(\frac{J}{F^{2}}\right)$ versus $\frac{1}{F}$, the data gives a straight line whose slope give access to the work function

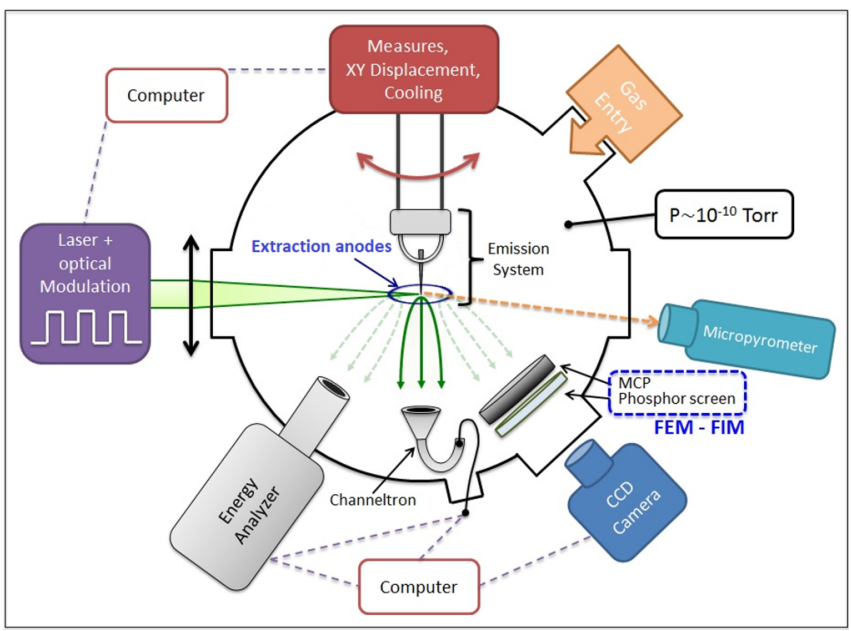

FIG. 4. Sketch of the experimental system used in this study. The sample is glued at the apex of a W tip which is spot welded on a $\mathrm{W}$ heating loop. Voltage is applied between the tip and a ringlike extraction anode ( $2 \mathrm{~mm}$ between tip and anode). The whole emission system can be cooled and displaced in order to send emitted electron toward various measurement devices, namely: energy analyser, electron counting, CCD camera. A micropyrometer is used to estimate the tip temperature during Joule heating. A laser (not used in this study) can be sent on the sample. Although the base vacuum is about $10^{-10}$ Torr, various gases can be injected in the chamber. 
$\Phi$, provided the electrical field $F$ is known. Deviations from straight line behavior occurs for many reasons, e.g., strong saturations in the emission current for semiconducting samples [33] or effect of series resistance [34].

Note that the local electric field $(F)$ is not simply the ratio of the voltage difference divided by the distance between the emitter and the electrode, the enhancement effect due to the shape of the usually sharp emitter (so called tip effect) has to be included. The local field $F$ is then linked to the macroscopic applied voltage $V$ via the relationship $F=\beta \mathrm{V}$. To extract $\beta$ from a straight F.-N. plot, one should know $\Phi$ and have already a fairly good approximation of the function $v(F)$. In our complex setup, the $\beta$ will include the enhancement due to the tip support, the width and length of the flake, the degree of roughness along the edge, the local roughness, the width of the graphene and finally the atomic structure and how it adjusts to these high local fields.

The total energy distribution (TED) of the emitted electrons measured directly by an electron energy analyzer as used in surface physics is given by: $j_{0}(E)=\frac{J_{0}}{e d_{0}} \frac{\exp \left(\frac{E-E_{F}}{d_{0}}\right)}{\left.1+\exp \frac{\left(E-E_{F}\right.}{k_{B} T}\right)}$, where $\frac{1}{d_{0}} \sim 1.025 \frac{\Phi^{1 / 2}}{F}$. The TED peak shape and position contain much basic information. In general for a simple metallic case a broadening on the high-energy side is a signature of a temperature increase, while a broadening on the low-energy side marks an increased electric field. The peak position for a metal is exactly at the Fermi level of the emission zone and to the first order at the voltage applied to the tip, but shifts occur if there are voltage drops leading up to the apex and also due to nanometric effects such as occur for quantum dots (QDs) as discussed below. Field emission is extremely sensitive to contamination that occurs even in ultrahigh vacuum. As well, the high electric fields that are typical for field emission $(\sim 5 \mathrm{~V} / \mathrm{nm})$ push the adsorbates to the high electric field zones largely amplifying the absorption in the emission zone. Besides simply changing the work function or slightly modifying the TEDs, they are driven further by field and local heating effect to agglomerate into high $\beta$ nanostructures that become QDs and the principal emitting sources [35]. Their formation is ubiquitous on uncleaned or poorly cleaned surface.

A critical point in this work is the difficulty to unambiguously distinguish this type of emission site from the true graphene edge and corner emitters, though we can "do our best" by careful in situ cleaning. The FE of these nanostructures differs from metallic surfaces with FN slope changes, apparition of new peaks related to quantum confinement in the TEDs, and field-induced shifts of the spectra. These characteristics can also evolve very rapidly with even a slight modification of the nanostructure. The basics of the model are described by a resonant tunneling process, as shown in Fig. 5. When increasing the extraction voltage, these states move linearly with the voltage because of the field penetration. When a state, previously above the Fermi level, reaches the Fermi level due to this field penetration, a new window for electrons emission opens, which causes a kink in the current-voltage characteristic and a new peak in the TED. The nanostructure TED peaks also have very low broadening on the low energy sides when increasing the field, in contrast to metals. Peaks widths typically vary from $0.1-2 \mathrm{eV}$.
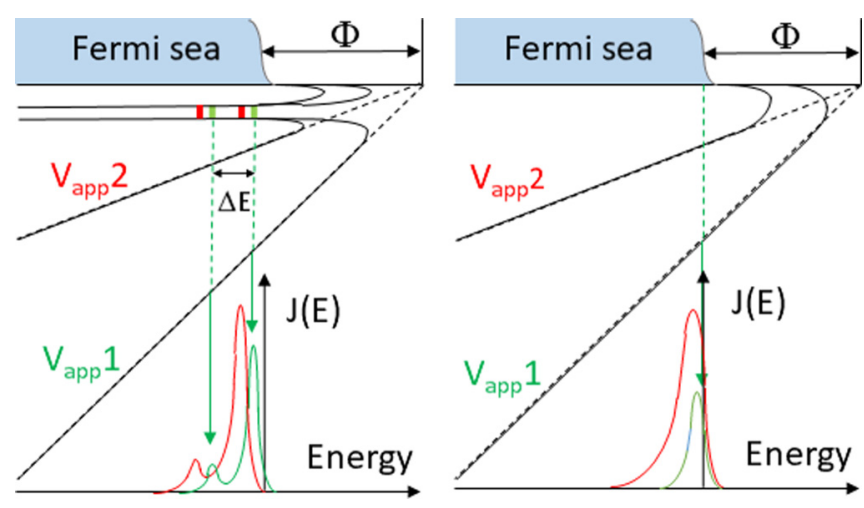

FIG. 5. Field emission from a metallic emitter (right) compared to an adsorbate (left) for nonzero temperature. For a metal with work function $\Phi$, the Fermi level fixes the TED maximum distribution position; only one peak is possible. Increasing the extraction field (from low voltage $V_{\text {app }} 1$, green peak to higher voltage $V_{\text {app }} 2$, red peak) yields in an increase of the emitted current and a broadening of the low energy side of the TED peak. In the presence of adsorbates, emission occurs via a resonant tunneling effect. The localized states in the adsorbate are separated from the main emitter Fermi sea by a thin tunnel barrier, then the localized state can emit in vacuum through the field controlled triangular tunnel barrier. The adsorbates act as quantum dots in which the energy levels $\Delta \mathrm{E}$ are separated by quantum confinement, tunneling through theses states lead to multiple peaks in the energy distribution as shown in this drawing. Contrarily to the metallic case, the position of these peaks will also shift with applied extraction voltage as their position is not fixed by the Fermi level.

\section{RESULTS}

We first study graphene field emission from a "pristine" sample, which means without any specific cleaning processing except for a moderate heating $\left(\sim 600^{\circ} \mathrm{C}\right)$ required to obtain a stable emission. The first FEM images consisted of one spot [Fig. 6(a)] presumably from the dominant nanostructure. A mechanical vibration signature test is performed: While our sample is emitting, a small AC voltage is scanned in frequency until an electromechanical resonance occurs, which is observable by an elongation of the FE spot [Fig. 6(b)]. As the emission pattern shape during mechanical vibration is vertically elongated, we infer that the graphene flake is almost horizontal within our system. We also checked that the graphene sheet electromechanical frequency can be electrostatically tuned [36], which helps determine it is indeed graphene that resonates and not some other system (tungsten tip or other adverse cavity effect). This test is also extremely useful to check that our sample is still alive after outgassing or other treatments involving a change of the emission sites. The resonance frequencies were in the $\mathrm{MHz}$ range throughout this work and are discussed in another publication [37].

To start the cleaning procedure, the sample was gradually heated up to $1000^{\circ} \mathrm{C}$, always under a FE extraction field. After thermal cleaning, mechanical resonance tests were performed to check that the graphene sample was still undamaged. The FEM images still consisted of one spot [Fig. 7(c)] but with a clear elliptical shape as expected for a edge emitter, which should electrostatically impose weaker field focusing 


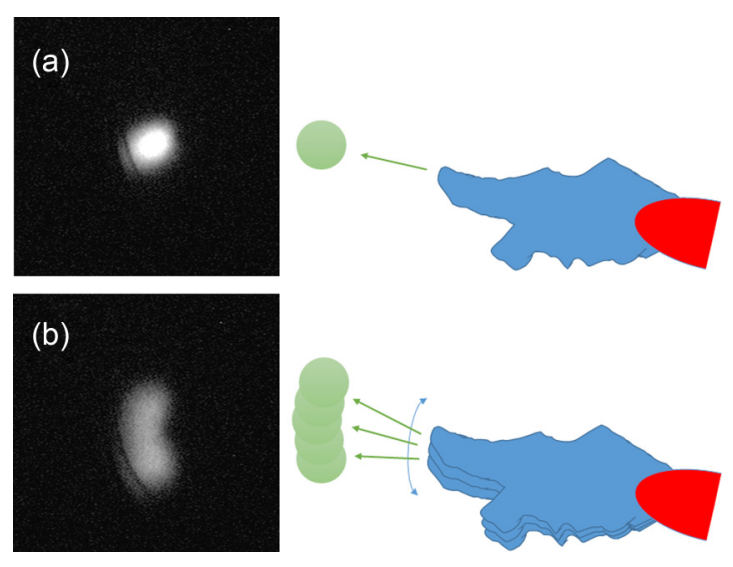

FIG. 6. (a) FE pattern observed when graphene sheet is out of mechanical resonance exhibiting a single emission spot. (b) When the graphene sheet enters in mechanical resonance, the pattern is spread vertically as the tip vibrates. As we expect the "easy vibration" first mode for a 2D object to be perpendicular to its plane, we can conclude that the graphene flake must be typically horizontal in our setup.

perpendicular to the edge (see discussion in Ref. [11]). Note that the elliptical deformation is consistent with the flake direction inferred from the mechanical resonance experiment
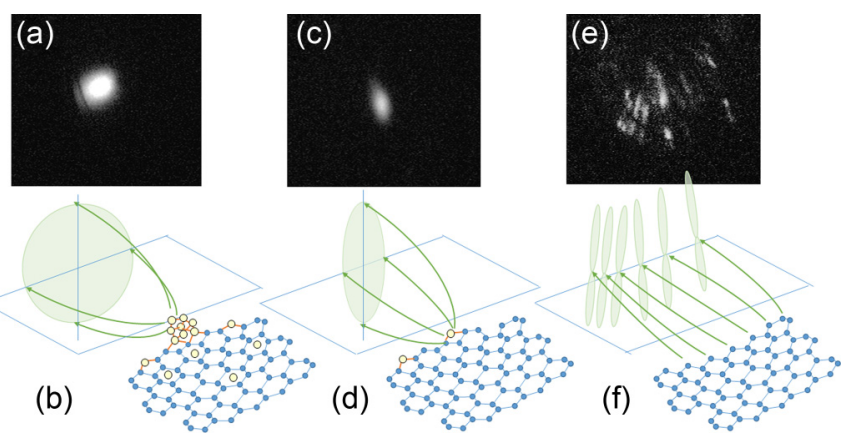

FIG. 7. (a) FE pattern from unprocessed dirty graphene dominated by a single emitter (nanoprotrusion). The nanoprotrusion consists usually of a bunch of atoms or molecules (white spheres on the sketch) with a conelike local environment which produces a symmetrical pattern (same vertical or horizontal lens effect) as described in the sketch (b). (c) FE pattern form thermally cleaned graphene. Thermal treatment removed foreign physiosorbed molecules but leaves graphene irregularities and strongly chemisorbed atoms. The emission is still dominated by a single emitter as there will always be a place with a local higher $\beta$. However, the local environment is no longer symmetric: The pattern is focused along the graphene edge direction but not on the perpendicular direction. As a consequence, the pattern is elliptical with main axis perpendicular to the graphene sheet (d). (e) Field ion microscopy (FIM) pattern from desorbed graphene using oxygen. As field desorption eliminates local asperities until the field is even, emission is no longer dominated by a single spot but multiple emitters are now visible. Note that the pattern of each emitter shows vertically elongated shapes with two wings separated by a darker center. The set of projected patterns are not perfectly aligned but follow the graphene edge local bending and crinkling as depicted in (f).

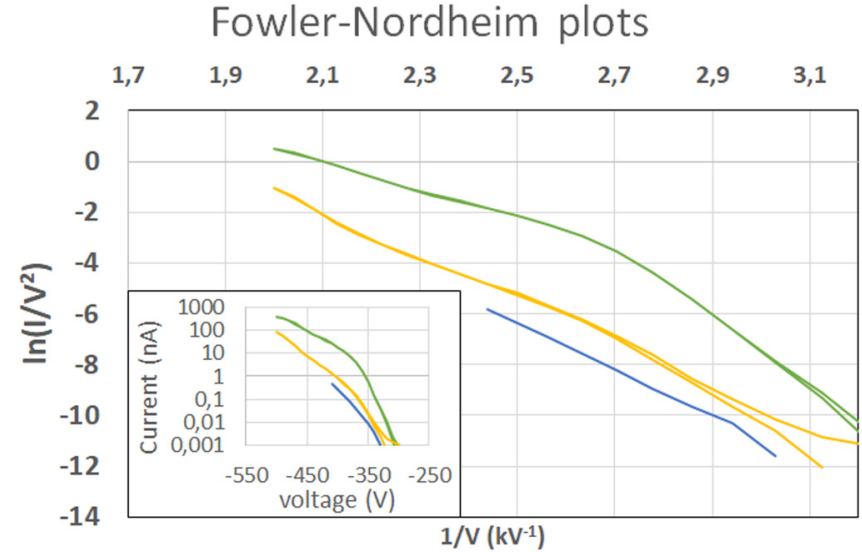

FIG. 8. Fowler-Nordheim curves on pristine graphene (top), after high temperature curing (middle) and after field desorption (bottom). These plots cover ranges of $300-500$ Volts for $160 \mathrm{pA}$ to $4 \mu \mathrm{A}$ range (five orders of magnitude in current). Inset: $\mathrm{I}(\mathrm{V})$ curve for the three sets of data, same color/order. Current is plotted on log scale.

and the SEM imaging of the sample before mounting in the FEM system.

To better eliminate these nanostructures, we used fieldinduced desorption techniques which is more local than thermal cleaning [38]. In short, we introduce $10^{-4}$ Torr of $\mathrm{O}_{2}$ in the chamber while applying a positive voltage to the tip until ionic field emission pattern is observed. Field desorption eliminates local asperities where the electric field is the highest. This process can be used to smooth nanowire ends up to atomic level [39]. As the first principal emitters are removed, new emission sites appear at higher voltage [see Fig. 7(e)]. Each emitter is elliptically shaped in the same direction as expected, although they are not perfectly aligned. This could be a consequence of local crinkling of the graphene sheet which will tilt the average trajectory of each emitter. It is worth noting that these kinds of emission patterns exhibiting two symmetric lobes with a darker central band have already been observed by Yokoyama et al. on multigraphene edges [11] and were attributed to edge state symmetry. In their work, Yokoyama et al. also observed that emission from an adsorbed molecule on the graphene edge is elliptical but deprived from the dark central band [as we observe in Fig. 7(c)] while emission from a bigger impurity loses the elongated property [as we observe in Fig. 7(a)].

The FN and TED curves were recorded during each cleaning stage. The TED data are accessible in Ref. [40]. The I/V characteristics were not the main thrust of this work but we present them for completeness because it sets the scale for voltage and current for other researchers.

The FN plots are presented in Fig. 8. Several changes in the slope can be seen as can be expected from a "QD" sample (Fig. 8 upper curve) as new energy level of the QD become available for emission (see Fig. 5). On the thermally cleaned sample, the plot is straighter and on the field desorbed sample perfectly straight though the measurement was carried out over a reduced range (Fig. 8 middle and lower curve).

The big uncertainty in proportionality between the pertinent field $F$ for tunneling and the applied voltage $V(F=\beta V)$ due the mounting on a tip support and the jagged form of 


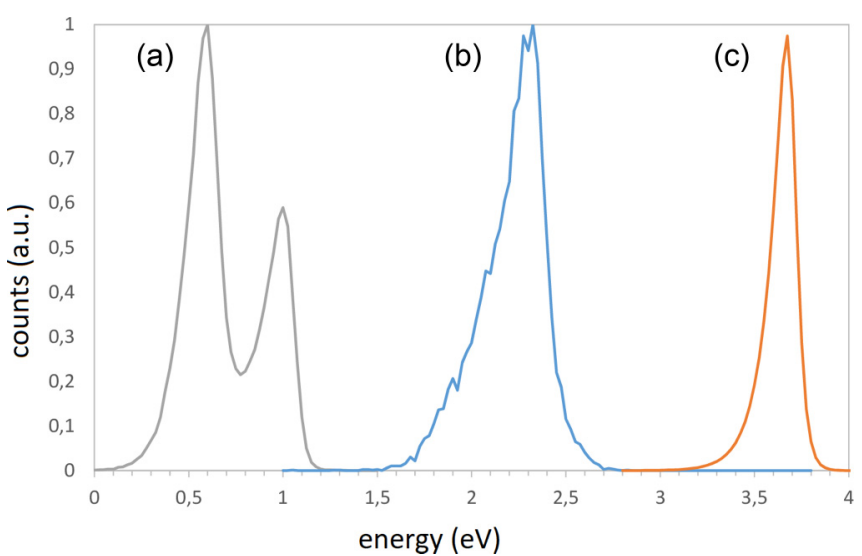

FIG. 9. Total energy distribution (TED) curves of (a) untreated, (b) heated, and (c) desorbed sample for comparable emission current (respectively, 7.5, 12, and $8 \mathrm{pA}$ ). FWHM are, respectively, 0.20 (highest peak), 0.26 , and $0.15 \mathrm{eV}$. Curves have been arbitrarily shifted for clarity.

the flake at many length scales means that it is extremely hazardous to interpret the FN slopes in terms of a local field enhancement at the emitting zone. As well different sections of the pristine and heated sample have quite different slopes. This is the source of major confusion in the field and we did not wish to repeat that error. In fact one should compare fits with other versions of the FN theory for edges [25] if the data merits such a treatment. However, for completeness, we have extracted the $\beta$ from our data from fits with basic FN theory with a nominal work function of $5 \mathrm{eV}$ and $v(F) \sim 0.7$ [41]. Results give a $\beta$ between 4 and $1010^{6} \mathrm{~m}^{-1}$ (pristine), 5.6 $10^{6} \mathrm{~m}^{-1}$ (heated), and $6.210^{6} \mathrm{~m}^{-1}$ (desorbed). One can be surprised that an atomic-thin-like structure does not exhibit a larger amplification factor, however electrostatics shows that a knife-edge geometric is less interesting than a needlelike structure (see Ref. [42] for discussion and calculation). The TED spectra of, respectively, pristine, heat treated, and desorbed graphene, are compared in Fig. 9. These three TED were recorded around $10 \mathrm{pA}$ of emission at room temperature. The multiple peaks feature produced by adsorbates (as explained in Fig. 5) visible on the untreated sample disappears after thermal curing. The TED full width at half maximum (FWHM) evolution is also interesting. While there is an adsorbate, the TED peaks are a rather narrow $(0.2 \mathrm{eV})$ consistent with localized state in the adsorbate that yields a set of narrow energy windows for electron emission (see Fig. 5). Once adsorbates are thermally removed, the electrons are emitted through a triangular barrier and the TED becomes slightly wider $(0.26 \mathrm{eV})$ with broadening on low energy side, signature of the field effect. Oddly enough, on the desorbed sample, the TED peaks FWHM shrinks down to $0.15 \mathrm{eV}$. One might immediately think of an adsorbate, but the absence of second peak on $1 \mathrm{eV}$ range means that the quantum dot should be extremely small and electrically insulated from the graphene sheet.

Cooling the sample holder with liquid air leads to a slightly narrower TED peak ( $0.13 \mathrm{eV}$ compared to $0.15 \mathrm{eV}$ for the room temperature emitter) at low current, consistent with a peak width reduction on the high-energy side. The tempera-

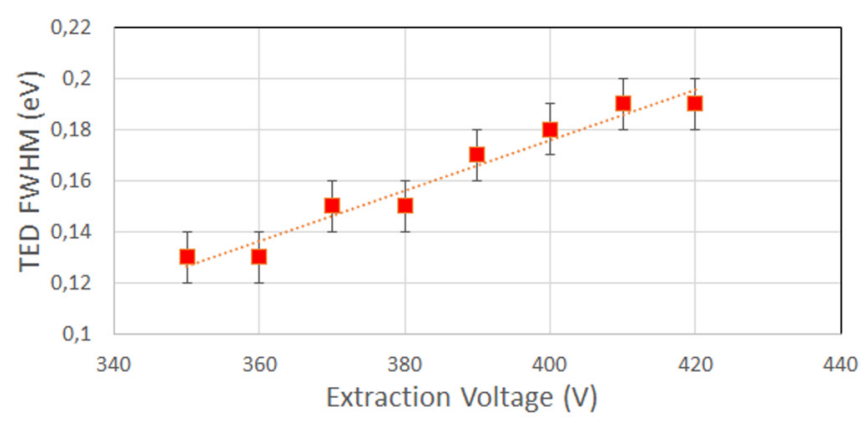

FIG. 10. Desorbed sample TED FWHM versus extraction voltage at low temperature. The linear width increase is expected as the triangular tunnel barrier is reduced by the increasing extraction field.

ture reduction was estimated [40] to be only about 60 to $70 \mathrm{~K}$ due to poor thermal conductivity of our setup. Our spectra are $\sim 2-3$ time narrower than a metallic emission peak, $\sim 15$ time narrower than previous measurements on graphene [13], six times narrower than one of the theoretical value [19] and 3 time larger than the other [20], which is surprisingly narrow at $\sim 0.06 \mathrm{eV}$, to our knowledge only once achieved for FE in any experiment [43]. A final point is that our individual peaks all have the asymmetric form characteristic of tunneling from a metal through a triangular barrier which is not the case of the theoretical prediction whose peaks do not appear to have a high energy temperature edge.

We now turn our attention toward the TED peak behavior with extraction voltage or emission current. For a clean metal at a given temperature, the Fermi level controls the TED position and one can observe a broadening on the low energy side with increased extraction voltage and/or a broadening on the high energy side with increasing temperature [31]. We indeed observe that TED FWHM increases linearly while increasing the extraction voltage (see Fig. 10). Although the TED is pinned to the Fermi level, the position of the TED peak maximum will slightly shift toward low energy because as the triangular barrier shrinks, more electrons are emitted in proportion below the Fermi level, thus shifting the peak maximum position. This shift is however extremely small and usually neglected (few meV over the full extraction field range, i.e. from 3 to $7 \mathrm{~V} / \mathrm{nm}$ ). It is however possible to observe a strong TED peak shift (several $\mathrm{eV}$ ) from a metallic emitter if it has a significant ohmic drop ( $\mathrm{M} \Omega$ range) as it was measured on CVD nanotubes [35]. In this case however, the TED peak shifts linearly with respect to the emission current. When emission is made through a quantum dot (adsorbate), the TED peak(s) are shifting linearly with respect to the extraction voltage as their energy level are shifted with the applied field (see Fig. 5). Measurements made on such samples [35] report a shift of $10 \mathrm{meV}$ per volt, linear with voltage.

For both our heat-treated and desorbed graphene sample, we observe a small TED peak shift, linear with the extraction voltage (see Fig. 11). The shift is clear although small with a slope close to $-1.8 \pm 0.1 \mathrm{meV} /$ volt.

First, we can rule out that ohmic drop can play any role in our sample: As explained above, TED peak maximum shift will be linear with emission current, not voltage. Moreover, to reach a 0.113 volt shift for $500 \mathrm{pA}$, the graphene sheet should 


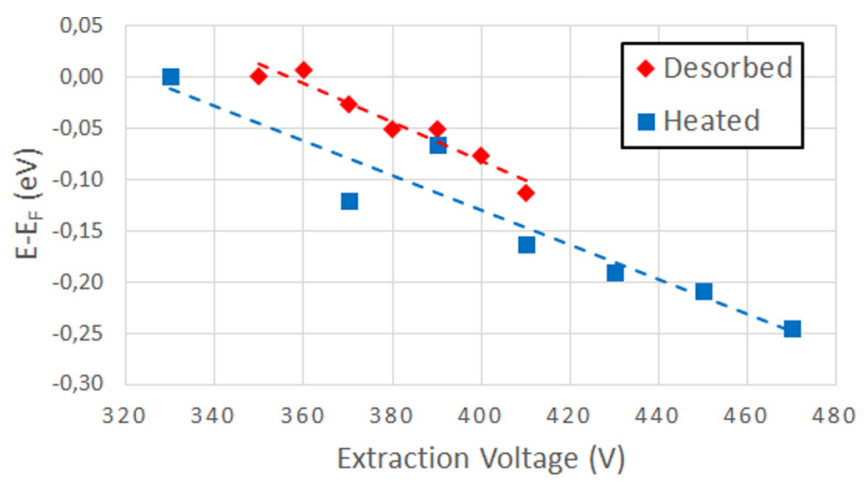

FIG. 11. TED peak position versus extraction voltage for heattreated sample (square) and desorbed sample (diamond). The peak position shift is linear with respect to extraction voltage. Measured slope are, respectively, $-1.7 \mathrm{meV} /$ volt (heated) and $-1.9 \mathrm{meV} /$ volt (desorbed).

have a $225 \mathrm{M} \Omega$ electric resistance which is clearly not what is expected for clean graphene.

Comparable small linear peak shift versus extraction voltage have already been observed on low current field emission from $\mathrm{SiC}$ nanowire with slope of $-2.2 \mathrm{meV} /$ volt (see Ref. [44], Fig. 4.34b, p. 135). As explained above, the voltage applied at the tip of the emitter deviates from the measured voltage if there is a voltage drop along the circuit. A short theoretical analysis [40] shows that on our limited voltage range explored $(\sim 200 \mathrm{~V})$, it is perfectly possible to observe such a small linear shift if there is a Schottky barrier at the graphene-W tip contact.

Finally, let's focus on the last difference between thermally treated and desorbed sample: As already mentioned, the desorbed TED peak is sensibly narrower than the heat treated. Note that the main effect resides on the low energy side of the TED peak which exact shape is controlled by the triangular tunnel barrier details. It is known from field emission studies that any surface imperfection will lead to deviation from the theoretical perfect triangular shape, usually increasing the
TED width. We can speculate that the field desorption has removed teared edges imperfections and what we observe now is the genuine graphene edge emission. We propose that the narrowness of the peak could be a direct manifestation of the curving of the barrier potential due to the atomic scale of the graphene edge which to first order is controlled by the slope of the tunnel barrier at the second crossing point where the potential crosses $E_{F}$. The measured FWHM implies that the field at $1 \mathrm{~nm}$ from the edge is about $1 / 2$ the surface value, in line with an atomic scale tip. A similar argument for energy filtering has been used to explain the narrow TEDs for $L a B_{6}$ nanowires, down to $0.2 \mathrm{eV}$, where the low slope at the $E_{F}$ crossing occurs because lower fields are necessary to induce emission on the low work function material [45].

\section{CONCLUSION}

We explore the preparation process of clean graphene sample for field emission studies. The sample characterization (TEM and Raman spectroscopy), cleaning procedure, ultrahigh vacuum environment, and mechanical vibration control strategy confirms that the measured object is indeed a carefully desorbed single layer graphene. Clean graphene shows a straight F.-N. behavior in our measurement range. Its TED peak is extremely narrow $(0.15 \mathrm{eV})$ at room temperature. There is a small linear peak shift with extraction voltage which is attributed to a Schottky barrier at the $\mathrm{W}$ tip contact. In conclusion we hope that these more controlled experiments and particularly the energy spectra can guide the scientific community to better understand emission from the graphene edge.

\section{ACKNOWLEDGMENTS}

The author acknowledges D. Guillot for realization of the TEM sample holder, N. Blanchard for technical support, and the CLYM (Centre Lyonnais de Microscopie) for the access to the microscope Topcon. This work was carried out within the framework of the Plateforme nanofils et nanotube Lyonnaise de l'Université Lyon 1.
[1] Z.-S. Wu, S. Pei, W. Ren, D. Tang, L. Gao, B. Liu, F. Li, C. Liu, and H.-M. Cheng, Field emission of single-layer graphene films prepared by electrophoretic deposition, Adv. Mater. 21, 1756 (2009).

[2] X.-P. Wang, L.-H. Liu, and L.-J. Wang, Diamond film, single layer carbon nanosheets film and diamond/carbon nanosheet composite film synthesis and field emission performance comparison, J. Alloys Compd. 727, 185 (2017).

[3] J. Meng, L. Zhangi, and Y. Fang, Damage to few-layer graphene sheets during electron field emission, Carbon 125, 370 (2017).

[4] X. Guo, S. Qin, S. Bai, H. Yue, Y. Li. Q. Chen, J. Li, and D. He, vertical graphene nanosheets synthesized by thermal chemical vapor deposition and the field emission properties, J. Phys. D: Appl. Phys. 49, 385301 (2016).

[5] R. T. Khare, V. Gelamo, M. A. More, D. J. Late, and C. S. Rout, Enhanced field emission of plasma treated multilayer graphene, Appl. Phys. Lett. 107, 123503 (2015).
[6] J. Liu, B. Zeng, W. Wang, N. Li, J. Guo, Y. Fang, J. Deng, J. $\mathrm{Li}$, and C. Hao, Graphene electron cannon: High-current edge emission from aligned graphene sheets, Appl. Phys. Lett. 104, 023101 (2014).

[7] S. Tang, Y. Zhang, N. Xu, R. Zhan, L. Gong, P. Zhao, J. Chen, C. Liang, J. Chen, J. She, and S. Deng, In situ study of graphene crystallinity effect on field electron emission characteristics, J. Vac. Sci. Technol. B 35, 02 C107 (2017)

[8] S. Santandrea, F. Giubileo, V. Grossi, S. Santucci, M. Passacantando, T. Schroeder, G. Lupina, and A. Di Bartolomeo, Field emission from single and few layer graphene flakes, Appl. Phys. Lett 98, 163109 (2011).

[9] Z. Xiao, J. She, S. Deng, Z. Tang, Z. Li, J. Lu, and N. Xu, Field electron emission characteristics and physical mechanism of individual single-layer graphene, ACS nano 4, 6332 (2010).

[10] K. Nakakubo, K. Asaka, H. Nakahara, and Y. Saito, Evolution of fiel electron emission pattern from multilayered graphene 
induced by structural change of edge, Appl. Phys. Express 5, 055101 (2012).

[11] N. Yokoyama, K. Nakakubo, K. Iwata, K. Asaka, H. Nakahara, and Y. Saito, Fied emission patterns showing symmetry of electronic states in graphene adges, Surf. Interface Anal. 48, 1217 (2016).

[12] G. N. Fursey, N. V. Egorov, I. I. Zakirov, A. M. Yafyasov, L. I. Antonova, and V. V. Trofimov, Peculiarities of the total energy distribution of field emission electrons from graphenelike structures, J. Commun. Technol. Electron. 61, 72 (2016).

[13] J. L. Shaw, J. B. Boos, B. D. Kong, J. T. Robinson, and G. G Jernigan, Field emission energy distribution and three-termial current-voltage characteristics from planar graphene edges, J. Appl. Phys 125, 054502 (2019).

[14] X. Shao, W. K. Ang, P. Balamuniappan, and A. Khursheed, Structural and electroni optimization of ring-graphene cathodes and their field emission properties, Appl. Phys. Lett. 114, 223101 (2019).

[15] J. T. H. Tsai, T. Y. E. Chu, J.-Y. Shiu, and C.-S. Yang, Field emission from individual freestanding graphene edge, Small 8 , 3739 (2012).

[16] L. Chen, H. Yua, J. Zhong, J. Wuc, and W. Su, Graphene field emitters: a review of fabrication, characterization and properties, Mater. Sci. Eng. B 220, 44 (2017).

[17] J. L. Lado, N. Garcia-Martinez, and J. Fernandez-Rossier, Edge states in graphene-like systems, Synthetic Metals 210, 56 (2015).

[18] Y. Shimomura, Y. Takane, and K. Wakabayashi, Electronic states and local density os states in graphene with a corner edge structure, J. Phys. Soc. Japan 80, 054710 (2011).

[19] K. Tada and K. Watanabe, Ab Initio Study of Field Emission from Graphitic Ribbons, Phys. Rev. Lett. 88, 127601 (2002).

[20] S. F. Huang, T. C. Leung, B. Li, and C. T. Chan, First-principle study of filed-emission properties of nanoscale graphite ribbon arrays, Phys. Rev. B 72, 035449 (2005).

[21] M. Luo and Z. Li, Multi-field electron emission pattern of 2D emitter: Illustrated with graphene, J. Appl. Phys 120, 204304 (2016).

[22] Z. Li, N. Xu, and H. J. Kreuzer, Coherent field emission image of graphene predicted with a microscopic theory, Phys. Rev. B 85, 115427 (2012).

[23] W. Wang, X. Qin, N. Xu, and Z. Li, Field electron emission characteristics of graphene, J. Appl. Phys. 109, 044304 (2011).

[24] Y. Gao and S. Okada, Field emission properties of edgefunctionalized graphene, Carbon 142, 190 (2019).

[25] X. Qin, W. Wang, N. Xu, Z. Li, and R. Forbes, Analytical treatment of cold field electron emission from a nanowall emitter, including quantum confinement effects, R. Soc. A 467, 1029 (2011).

[26] R. D. Young and E. W. Muller, Experimental measurement of the total-energy distribution of field-emitted electrons, Phys. Rev. 113, 115 (1959).

[27] S. T. Purcell, P. Vincent, C. Journet, and V. T. Binh, Hot Nanotube: Stable Heating of Individual Multiwalled Carbon Nanotubes to $2000 \mathrm{~K}$ Induced by the Field-Emission Current, Phys. Rev. Lett. 88, 105502 (2002).

[28] S. Choubak, M. Biron, P. L. Levesque, R. Martel, and P. Desjardins, No graphene etching in purified hydrogen, J. Phys. Chem. Lett. 4, 1100 (2013)
[29] S. Choubak, P. L. Levesque, E. Gaufres, M. Biron, P. Desjardins, and R. Martel, Graphene CVD: Intreplay between growth and etching on morphology and stacking by hydrogen and oxidizing impurities, J. Phys. Chem. C 118, 21532 (2014).

[30] P. Poncharal, A. Ayari, T. Michel, and J.-L. Sauvajol, Raman spectra of misoriended bilayer graphene, Phys. Rev. B 78, 113407 (2008).

[31] R. H. Fowler and L. Nordheim, Electron emission in intense electric fields, Royal Society of London Proceeding Series A 119, 173 (1928).

[32] F. R. Abbott and J. E. Henderson, The range and Validity of the Field Current Equation, Phys. Rev. 56, 113 (1939).

[33] M. Choueib, A. Ayari, P. Vincent, M. Bechelamy, D. Cornu, and S. T. Purcell, Strong deviations from Fowler-Nordheim behavior for field emission from individual $\mathrm{SiC}$ nanowires due to restricted bulk carrier generation, Phys. Rev. B 79, 075421 (2009).

[34] A. Di Bartolomeo, A. Scarfato, F. Giubileo, F. Bobba, M. Biasiucci, A. M. Cucolo, S. Santucci, and M. Passacantando, A local field emission study of partially aligned carbon-nanotubes by atomic force microscope probe, Carbon 45, 2957 (2007).

[35] S. T. Purcell, P. Vincent, M. Rodriguez, C. Journet, S. Vignoli, D. Guillot, and A. Ayari, Evolution of the field-emission properties of individual multiwall carbon nanotubes submitted to temperature and field treatments, Chem. Vap. Deposition 12, 331 (2006).

[36] S. T. Purcell, P. Vincent, C. Journet, and V. T. Binh, Tuning of Nanotube Mechanical Resonances by Electric Field Pulling, Phys. Rev. Lett. 89, 276103 (2002).

[37] A. Descombin, P. Poncharal, A. Pascale-Hamri, M. Choueib, R. Diehl, P. Vincent, ST. Purcell, A. Ayari, and S. Perisanu, Giant, voltage tuned, quality factors of single wall carbon nanotubes and graphene at room temperature, Nano Lett. 19, 1534 (2019).

[38] E. W. Muller and T. T. Tsong, Field Ion Microscopy, principles and applications (Elsevier, New York, 1969).

[39] P. Poncharal, P. Vincent, J. M. Benoit, S. Perisanu, A. Ayari, M. Choueib, and S. T. Purcell, Field evaporation tailoring of nanotubes and nanowires, Nanotechnology 21, 215303 (2010).

[40] See Supplemental Material at http://link.aps.org/supplemental/ 10.1103/PhysRevB.102.035416 for voltage drop model, emitter temperature, TED data \& sample image.

[41] A. Modinos, Field thermionic, and secondary electron emission spectroscopy (Plenum Publishing corporation, New York, 1984).

[42] S. T. Purcell, P. Vincent, S. Perisanu, A. Ayari, and P. Poncharal, Field emission from the edges of single layer graphene, in Nanostructured Carbon Electron Emitters and its Applications, edited by Y. Saito (Pan Stanford Publishing, Singapore, 2020).

[43] S. T. Purcell, V. T. Binh, and N. Garcia, $64 \mathrm{meV}$ measurement energy distribution from cold field emission nanotips, Appl. Phys. Lett. 67, 436 (1995).

[44] M. Choueib, Etude des propriete physique de nanofils individuels de Carbure de Silicium par emission de champ, Doctoral dissertation, Université Claude Bernard, Lyon 2009, available from https://tel.archives-ouvertes.fr/tel-00656991.

[45] H. Zhang, J. Tang, J. Yuan, Y. Yamauchi, T. T. Suzuki, N. Shinya, K. Nakajima, and L.-C. Qin, An ultrabright and monochromatic electron point source made of a $\mathrm{LaB}_{6}$ nanowire, Nat. Nanotechnol. 11, 273 (2015). 